\title{
Abbreviations in Bibliographical and Textual Material
}

\section{ABoT \\ AM \\ AnSt \\ $A O A T$ \\ AÖAW \\ AoF \\ ArAn \\ BiOr \\ BLMJ}

Boğ. III

BoHa 14

BoHa 22

BoHa 23

CAD

CHD

ChS

$\operatorname{CoS}$

$\mathrm{CTH}$

$D B H$

EA

Emar IV

Emar VI

Eothen

FHL

FsAlp

FsArchi

FsBeckman
Ankara arkeoloji müzesinde bulunan Boğazköy tabletleri

Goetze, Albrecht. Die Annalen des Muršiliš, MDOG 38. Leipzig: Hinrichs, 1933. Anatolian Studies (Journal of the British Institute of Archaeology at Ankara)

Alter Orient und Altes Testament

Anzeiger den Österreichischen Akademie der Wissenschaften. Phil-hist. Klasse Altorientalische Forschungen

Archivum Anatolicum

Bibliotheca Orientalis

Bible Lands Museum, Jerusalem

Bittel, Kurt et al. Boğazköy III. Funde aus den Grabungen 1952-1955. Berlin, 1957.

Boehmer, Rainer M. and Hans Gustav Güterbock. Glyptik aus dem Stadtgebiet von Boğazköy. Grabungskampagnen 1931-1939, 1952-1978, Berlin: Gebr. Mann, 1987.

Dinçol, Ali M. and Belkıs Dinçol. Die Prinzen- und Beamtensiegel aus der Oberstadt von Boğazköy-Hattusa von 16. Jahrhundert bis zum Ende der Grossreichzeit, Mainz am Rhein: von Zabern, 2008.

Herbordt, Suzanne, Daliah Bawanypeck, and J. David Hawkins. Die Siegel der Grosskönige und Grossköniginnen auf Tonbullen aus dem Nişantepe-Archiv in Hattusa, Mainz am Rhein: von Zabern, 2011.

The Assyrian Dictionary of the Oriental Institute of the University of Chicago

The Hittite Dictionary of the Oriental Institute of the University of Chicago

Corpus der hurritischen Sprachdenkmäler

Context of Scripture, vol. I-III, edited by W. W. Hallo. Leiden: Brill, 1997-2003. Laroche, Emmanuel. Catalogue des textes Hittites. Paris: Klincksieck, 1971.

Dresdner Beiträge zur Hethitologie

Die El-Amarna Tafeln, edited by J. A. Knudtzon. Lepzig, 1915.

Beyer, Dominique. Emar IV: Les sceaux. OBO Series Archaeologica 20. Göttingen: Vandenhoeck and Ruprecht, 2001.

Arnaud, Daniel. Recherches au pays d'Aštata. Textes sumériens et accadiens, Emar VI/1-4. Paris: Éditions Recherche sur les Civilisations, 1986.

Collana di studi sulle civiltà dell'Oriente antico. Firenze: LoGisma.

Durand, Jean-Marie and Emmanuel Laroche, Fragments hittites du Louvre, in Mémorial Atatürk: Études d'archéologie et de philologie anatoliennes, Institut Français d'études anatoliennes: Éditions Recherche sur les civilisations: Synthese 10. Paris, 1982, 73-107.

Hittite and Other Anatolian and Near Eastern Studies in Honor of Sedat Alp, edited by H. Otten, H. Ertem, E. Akurgal, and A. Süel. Ankara: TTK, 1992.

Studi di Ittitologia in onore di Alfonso Archi, edited by R. Francia and G. Torri. Orientalia 79/2. 2010.

Beyond Hatti: A Tribute to Gary Beckman, edited by B. J. Collins and P. Michalowski. Atlanta: Lockwood Press, 2013. 
FsCarruba

FsdeRoos

FsDinçol

FsGüterbock

FsGüterbock ${ }^{2}$

FsHaas

FsHawkins

FsHoffner

FsHouwink

ten Cate

FsKošak

FsLebrun II

FsMeriggi ${ }^{2}$

FsNeve

FsNÖzgüç

FsTÖzgüç

FsOtten ${ }^{2}$

FsPopko

FsTischler
Studi di Ittitologia in onore di Onofrio Carruba, edited by A. Archi and F. Pecchioli Daddi. OrNS 73/4. Rome: Pontificium Institutum Biblicum, 2004.

The Life and Times of Hattušili III and Tudhaliya IV. Proceedings of a Symposium Held in Honour of J. de Roos, 12-13 December 2003, Leiden, edited by Th. P. J. van den Hout. Leiden: Brill, 2006.

Belkıs Dinçol ve Ali Dinçol'a Armağan VITA Festschrift in Honor of Belkıs Dinçol and Ali Dinçol, edited by M. Alparslan, M. Doğan-Alparslan and H. Peker. Istanbul: Ege, 2007.

Anatolian Studies Presented to Hans Gustav Güterbock on the Occasion of his $65^{\text {th }}$ Birthday, edited by K. Bittel, Ph. H. J. Houwink ten Cate and E. Reiner. Istanbul: Nederlands Historisch-archaeologisch instituut te Istanbul, 1974.

Kaniššuwar: A Tribute to Hans G. Güterbock on his Seventy-fifth Birthday May 27, 1983, edited by H. A. Hoffner and G. M. Beckman. AS 23. Chicago: Oriental Institute, 1986.

Kulturgeschichten. Altorientalistische Studien für Volkert Haas zum 65. Geburtstag, edited by T. Richter, D. Prechel, and J. Klinger. Saarbrücken: Saarbrücker Druckerei und Verlag, 2001.

Ipamati kistamati pari tumatimis: Luwian and Hittite Studies presented to J. David Hawkins on the occasion of his 70th birthday, edited by I. Singer. TelAviv: Institute of Archaeology, 2010.

Hittite Studies in Honor of Harry A. Hoffner Jr. on the Occasion of His 65th Birthday, edited by G. Beckman, R. Beal, and G. McMahon. Winona Lake, IN: Eisenbrauns, 2003.

Studio Historiae Ardens. Ancient Near Eastern Studies Presented to

Philo H. J. Houwink ten Cate on the Occasion of his $65^{\text {th }}$ Birthday, edited by Th. P. J. van den Hout \& J. de Roos. Leiden: Nederlands Instituut vor het Nabije Oosten, 1995.

Tabularia Hethaeorum; Hethitologische Beiträge Silvin Košakzum 65. Geburtstag, edited by D. Groddek and M. Zorman. DBH 25. Wiesbaden: Harrassowitz, 2007.

Studia Anatolica et Varia. Mélanges offerts au Professeur René Lebrun (Vol. II), edited by M. Mazoyer and O. Casabonne. Paris: L'Harmattan, 2004.

Studia mediterranea: Piero Meriggi dictata, edited by 0. Carruba. Pavia: Aurora Edizioni, 1979.

Festschrift für Peter Neve, zum 65. Geburtstag am 3. April 1994 von Freunden und Kollegen, edited by J. Seeher. IstMitt 43. 1993.

Nimet Özgüç'e Armağan. Aspects of Art and Iconography: Anatolia and its Neighbors. Studies in Honor of Nimet Özgüç, edited by M. J. Mellink, E. Porada, and T. Özgüç. Ankara: TTK, 1993.

Anatolia and the Ancient Near East: Studies in Honor of Tahsin Özgüç, edited by K. Emre, B. Hrouda, M. J. Mellik, and N. Özgüç. Ankara: TTK, 1989.

Documentum Asiae minoris antiquae. Festschrift für Heinrich Otten zum 75. Geburtstag, edited by E. Neu and C. Rüster. Wiesbaden: Harrassowitz, 1988.

Silva Anatolica: Anatolian Studies Presented to M. Popko on the Occasion of His $65^{\text {th }}$ Birthday, edited by P. Taracha. Warsaw: Agade, 2002.

Anatolica et Indogermanica: studia linguistica in honorem Johannis Tischler septuagenarii dedicata, edited by H. Marquardt, S. Reichmuth, J. V. Garcia 
FsWilhelm

GsForrer

GsDiakonoff

GsImparati

GsNeu

HCCT-E

HED

HEG

HHCTO

HKM

$\mathrm{HW}^{2}$

$H Z L$

IBoT

ICH 1

ICH 2

$\mathrm{ICH} 3$

ICH 4

ICH 5

ICH 6

ICH 7
Trabazo. Innsbruck: Institut für Sprachen und Literaturen der Universität Innsbruck, 2016.

Festschrift für Gernot Wilhelm anlässlich seines 65. Geburtstages am 28. Januar 2010, edited by J. C. Fincke. Dresden: Islet, 2009.

Šarnikzel. Hethitologische Studien zum Gedenken an Emil Orgetorix Forrer, edited by D. Groddek und S. Rößle. DBH 10. Dresden: TU Dresden, 2004.

Memoriae Igor M. Diakonoff, edited by L. Kogan, N. Koslova, S. V. Loesov, and S.Tishchenko. Winona Lake, IN: Eisenbrauns, 2005.

Anatolia antica. Studi in memoria di Fiorella Imparati, edited by S. de Martino and F. Pecchioli Daddi. Eothen 11. Firenze: LoGisma, 2002.

Investigationes Anatolicae: Gedenkschrift für Erich Neu, edited by J. Klinger, E. Rieken, and C. Rüster. StBoT 52. Wiesbaden: Harrassowitz, 2010.

Hirayama Collection Cuneiform Texts (in Japan), Emar.

Puhvel, Jaan. Hittite Etymological Dictionary. Berlin - New York: Mouton, $1984 \mathrm{ff}$.

Tischler, Johann. Hethitisches etymologisches Glossar, Innsbruck: Inst. für Sprachen und Literaturen der Univ. Innsbruck, 1977 ff.

Ünal, Ahmet. Hittite and Hurrian Cuneiform Tablets from Ortaköy (Çorum), Central Turkey. Istanbul: Simurg, 1998.

Alp, Sedat. Hethitische Keilschrifttafeln aus Maşat. Ankara: TTK, 1991.

Friedrich, Johannes, A. Kammenhuber and I. Hoffmann, Hethitisches Wörterbuch. 2nd ed. Indogermanische Bibliothek. 2. Reihe: Wörterbücher. Heidelberg: Carl Winter, $1975 \mathrm{ff}$.

Rüster, Christel and Erich Neu. Hethitisches Zeichenlexikon: Inventar und Interpretation der Keilschriftzeichen aus den Boğazköy-Texten, (StBoTB 2). Wiesbaden: Harrasowitz, 1989.

Istanbul Arkeoloji Müzelerinde Bulunan Boğazköy Tabletleri 1-4. Istanbul Ankara: TTK, 1991.

Uluslararası 1. Hititoloji Kongresi Bildirileri (19-21 Temmuz 1990). Çorum, Ankara: Uluslararası Çorum Hitit Festivali Komitesi, 1992.

Atti del Il congresso internazionale di hittitologia, Pavia 28 giugno - 2 luglio 1993, edited by O. Carruba, M. Giorgieri, and C. Mora. StMed 9. Pavia: Gianni Iuculano, 1995.

III. Uluslararası Hititoloji Kongresi Bildirileri, Çorum 16-22 Eylül 1996 - Acts of the IIIrd International Congress of Hittitology, Çorum, September 16-22, 1996, edited by S. Alp and A. Süel. Ankara: Uyum Ajans, 1998.

Akten des IV. Internationalen Kongresses für Hethitologie, Würzburg, 4-8 Oktober 1999, edited by G. Wilhelm. StBoT 45. Wiesbaden: Harrassowitz, 2001. V. Uluslararası Hititoloji Kongresi Bildirileri, Çorum 02-08 Eylül 2002 - Acts of the Vth International Congress of Hittitology, Çorum September 02-08, 2002, edited by A. Süel. Ankara: Nokta Ofset, 2005.

VI Congresso Internazionale di Ittitologia. Roma, 5-9 settembre 2005. Parte I, (SMEA 49), Parte II, (SMEA 50), edited by A. Archi and R. Francia. Rome: CNR, Istituto di studi sulle civiltà dell'Egeo e del Vicino Oriente, 2007-2008.

VII. Uluslararası Hititoloji Kongresi Bildirileri Çorum 25-31 Ağustos 2008-Acts of the VIIth International Congress of Hittitology Çorum, August 25-31, 2008, edited by A. Süel. Ankara: Anıt, 2010. 
$\mathrm{ICH} 8$

VIII. 2011. Proceedings of the Eighth International Congress of Hittitology. Warsaw, 5-9 September, 2011, edited by P. Taracha. Warsaw: Wydawnictwo Agade, 2014.

JANES Journal of the Ancient Near Eastern Society of Columbia University

JAOS Journal of the American Oriental Society

JCS

$J E O L$ Journal of Cuneiform Studies

$J K F$ Jaarbericht van het Voor-Aziatisch-Egyptisch-Gezelschap. Ex Oriente Lux

JNES Jahrbuch für Kleinasiatische Forschung (= Anadolu Araştırmaları)

KBo Journal of Near Eastern Studies

Konkordanz Keilschrifttexte aus Boghazköi. Leipzig - Berlin

KTU

KUB

KuSa Košak, Silvin. Konkordanz der hethitischen Keilschrifttafeln. In http:// hethiter. net/hetkonk/ (v. 1.95).

The Cuneiform Alphabetic Texts from Ugarit, Ras Ibn Hani and Other Places, edited by M. Dietrich, O. Loretz, and J. Sanmartín. $2^{\text {nd }}$ ed. Münster: Ugarit Verlag, 1995.

Keilschrifturkunden aus Boghazköi. Berlin

Wilhelm, Gernot. Keilschrifttexte aus Gebäude A (Kuşaklı - Sarissa. Band 1: Keilschrifttexte, Faszikel 1) - Rahden: Verlag Marie Leidorf, 1997.

LhK Rüster, Christel and Gernot Wilhelm. Landschenkungsurkunden hethitischer Könige, StBoT Beiheft 4, Wiesbaden: Harrassowitz, 2012.

MDOG

$M I O$ Mitteilungen der Deutschen Orient-Gesellschaft zu Berlin. Berlin

Mora $[\mathrm{x}]$ Mitteilungen des Instituts für Orientforschung. Berlin

Mora, Clelia. La Glittica Anatolica del II Millennio A.C.: Classificazione Tipologica, StMed 6. Pavia: Gianni luculano, 1987.

Mitteilungen der Vorderasiatisch-ägyptischen Gesellschaft. Leipzig

Laroche, Emmanuel. Les Noms des Hittites, Études linguistiques 4, Paris: Klincksieck, 1966, and “Les noms des Hittites: supplément,” Hethitica 4. 1981, 3-58.

Niş Herbordt, Suzanne. Die Prinzen- und Beamtensiegel der hethitischen Grossreichszeit auf Tonbullen aus dem Nişantepe-archiv in Hattusa, BoHa 19. Mainz am Rhein: von Zabern, 2005.

$O A$

Oriens Antiquus. Rome

$O L Z$

OrNS

Orientalistische Literaturzeitung. Berlin

PRU III

Orientalia New Series. Rome

Nougayrol, Jean. Le palais royal d'Ugarit III: Textes accadiens at hourrites des archives est, ouest et centrales. Paris: Klincksieck, 1955.

PRU IV Nougayrol, Jean. Le palais royal d'Ugarit IV: Textes accadiens des archives sud. Paris: Klincksieck, 1956.

PRU V Virolleaud, Charles. Le palais royal d'Ugarit V: Textes alphabetiques des archives sud, sud-ouest du petit palais. Paris: Klincksieck, 1965.

PRU VI Nougayrol, Jean. Le palais royal d'Ugarit VI: Textes en cunéiformes babyloniens des archives du grand palais et du palais sud d'Ugarit. Paris: Klincksieck, 1970.

$R A$ Revue d'Assyriologie et d'Archéologie orientale. Paris

RANT

$R E$ Res Antiquae. Bruxelles.

Beckman, Gary. Texts from the Vicinity of Emar in the Collection of Jonathan Rosen, HANE/M 2, Padova: Sargon, 1996. 
RGTC

RHA

RIMA 1

RIA

RSO VII

RSO XXIII

SBo I

SBo II

SMEA

StAs

StBoT

StBoTB

StMed

Tel Aviv

THeth

TTK

UF

Ugar. III

Ugar. V

VBoT

VS NF

WO

WVDOG

$Z A$
Répertoire Géographique des Textes Cunéiformes. Wiesbaden

Révue Hittite et Asianique. Paris

Royal Inscriptions of Mesopotamia, Assyrian Periods v. 2: Assyrian Rulers of the Third and Second Millennia BC. Toronto, 1987.

Reallexikon der Assyriologie (und Vorderasiatischen Archäologie). Berlin

Une bibliothèque au sud de la ville: Les textes de la 34 campagne (1973), edited by P. Bordreuil. Paris: Editions Recherche sur les Civilisations, 1991.

Lackenbacher, Sylvie and Florence Malbran-Labat. 2016. Letters en Akkadien de la “Maison d'Urtenu:" Fouilles de 1994. Leuven: Peeters, 2016.

Güterbock, Hans Gustav. Siegel aus Boğazköy I (AfO Beiheft 5). Berlin: Selbstverlag, 1940.

Güterbock, Hans Gustav. Siegel aus Boğazköy II (AfO Beiheft 7). Berlin: Selbstverlag, 1942.

Studi micenei ed egeo-anatolici. Rome

Studia Asiana, Rome

Studien zu den Boğazköy-Texten. Wiesbaden

Studien zu den Boğazköy-Texten: Beihefte. Wiesbaden

Studia Mediterranea. Pavia: Gianni luculano

Journal of the Institute of Archaeology of Tel Aviv University. Tel-Aviv

Texte der Hethiter. Heidelberg

Türk Tarih Kurumu. Ankara

Ugarit-Forschungen. Kevelaer

Schaeffer, Cl. F.-A. et al., Ugaritica III. Sceaux et cylindres hittites, épée gravée du cartouche de Mineptah. tablettes chypro-minoennes et autres découvertes nouvelles da ras shamra. Paris: Klincksieck, 1956.

Schaeffer, Cl. F.-A. et al., Ugaritica V. Nouveaux textes accadiens, hourrites et ugaritiques des archives et bibliothèques privées d'Ugarit; commentaires des textes historiques (première partie). Paris: Klincksieck, 1968.

Götze, Albrecht. Verstreute Boghazköi-Texte. Marburg, 1930.

Jakob-Rost, Liane. Keilschrifttexte aus Boghazköy im Vorderasiatischen Museum (Vorderasiatische Schriftdenkmäler der Staatlichen Museen zu Berlin, Neue Folge, Heft XII). Mainz am Rhein: von Zabern, 1997.

Die Welt des Orients

Wissenschaftliche Veröffentlichungen der Deutschen Orient-Gesellschaft. Leipzig - Berlin

Zeitschrift für Assyriologie und verwandte Gebiete. Leipzig - Berlin 